\title{
Prevalência de anomalias dentárias em radiografias panorâmicas
}

\author{
Prevalence of dental anomalies in panoramic radiographies \\ Prevalencias de anomalías dentales en radiografías panorámicas \\ Roque Soares MARTINS NETO ${ }^{1}$ \\ Ivna Freitas de Sousa ALVES ${ }^{2}$ \\ Arthur Lima MACHADO ${ }^{2}$ \\ Luiz Alves BARBOSA NETO² \\ Andressa Aires ALENCAR ${ }^{3}$ \\ Diego Felipe Silveira ESSES ${ }^{4}$ \\ ${ }^{l}$ Residente em Cirurgia e Traumatologia Buco-Maxilo-Facial do Hospital Universitário Presidente Dutra da Universidade Federal do Maranhão HUPD-UFMA, \\ 65020-070 São Luís-MA, Brasil \\ ${ }^{2}$ Cirurgião-Dentista pelo Centro Universitário Católica de Quixadá - UNICATÓLICA, 63900-000 Quixadá - CE, Brasil \\ ${ }^{3}$ Residente em Saúde da Família e Comunidade pela Residência Integrada em Saúde da Escola de Saúde Pública do Ceará, 60165-090 Fortaleza - CE, Brasil \\ ${ }^{4}$ Mestre em Cirurgia e Traumatologia Buco-Maxilo-Facial pela Universidade Federal do Ceará, 60430-160 Fortaleza-CE, Brasil
}

\section{Resumo}

Introdução: As anomalias de desenvolvimento dentário ocorrem devido a distúrbios que acontecem durante a formação e a diferenciação celular. Dentre as radiografias odontológicas, a panorâmica se destaca por ser um exame radiográfico mais abrangente, que auxilia no diagnóstico e no planejamento terapêutico dos processos patológicos dos dentes e dos ossos da face. Objetivo: O objetivo desse estudo é determinar a prevalência de anomalias dentárias em radiografias panorâmicas de pacientes em tratamento no Centro Universitário Católica de Quixadá-CE e no Centro de Especialidades Odontológicas de Quixeramobim-CE. Métodos: A análise radiográfica foi realizada por um único pesquisador e os achados radiográficos foram escritos em uma ficha devidamente desenvolvida para este estudo. Resultados: Foram analisadas 500 radiografias panorâmicas de pacientes que possuíam entre 5 e 50 anos de idade. Desses, $67 \%$ (n=333) pertenciam ao sexo feminino e 33\% ( $\mathrm{n}=167)$ ao masculino. Foi encontrado um total de 1150 anomalias, correspondentes a: dentes não irrompidos $41,7 \%$ $(n=480)$, giroversão $24,4 \%(n=281)$, dilaceração radicular 20,3\% $(n=234)$, microdontia $6,7 \% \quad(n=77)$, agenesia 3,9\% ( $n=45)$, dentes supranumerários $2,4 \%(\mathrm{n}=28)$, taurodontia $0,3 \%(\mathrm{n}=4)$ e a macrodontia $0,1 \%(\mathrm{n}=1)$. As radiografias também foram divididas pelo tipo de arcada ocorrendo $48 \%(\mathrm{n}=307)$ na maxila e $52 \%(\mathrm{n}=330)$, na mandíbula. As anomalias dentárias hiperplasiantes foram encontradas em $2,9 \%$ $(n=33)$ da amostra, as hipoplasiantes em 10,6\% $(n=122)$ e as heterotópicas em 86,5\% (n=995). Desta forma conclui-se que na população estudada a maior prevalência correspondeu ao sexo feminino, à anomalia de desenvolvimento heterotópica e ao dente não irrompido.

Descritores: Anormalidades Dentárias; Radiografia; Radiografia Panorâmica.

\section{Abstract}

Introduction: Dental developmental anomalies occur due to disorders that occur during formation and cell differentiation. Among the dental radiographs, the panoramic view stands out as a more comprehensive radiographic examination, which assists in the diagnosis and therapeutic planning of the pathological processes of teeth and facial bones. Objective: The objective of this study is to determine the prevalence of dental anomalies in panoramic radiographs of patients undergoing treatment at the Catholic University of Quixadá-CE and the Quixeramobim-CE Center for Dental Specialties. Methods: The radiographic analysis was performed by a single investigator and the radiographic findings were written in a form properly developed for this study. Results: 500 panoramic radiographs were analyzed of patients who were between 5 and 50 years of age. Of these, $67 \%(n=333)$ were female and $33 \%(n=167)$ were male. A total of 1150 anomalies were found, corresponding to: unbroken teeth $41.7 \%(\mathrm{n}=480)$, rotation $24.4 \%(\mathrm{n}=281)$, root laceration $20.3 \%(\mathrm{n}=234)$, microdontia $6,7 \%$ $(n=77)$, agenesis $3.9 \%(n=45)$, supernumerary teeth $2.4 \%(n=28)$, taurodontia $0.3 \%(n=4)$ and macrodontia $0.1 \%=1)$. The radiographs were also divided by the type of arcade occurring $48 \%(\mathrm{n}=307)$ in the maxilla and $52 \%(\mathrm{n}=330)$, in the mandible. Hyperplastic dental anomalies were found in $2.9 \%(\mathrm{n}=33)$ of the sample, hypoplastic in $10.6 \%(\mathrm{n}=122)$ and heterotopic in $86.5 \%(\mathrm{n}=995)$. In this way, it was concluded that in the population studied the greatest predominance was female gender, the anomaly of heterotopic development and the nonerupted tooth.

Descriptors: Tooth Abnormalities; Radiography; Radiography, Panoramic.

\section{Resumen}

Introducción: Las anomalías de desarrollo dentario ocurren debido a disturbios que ocurren durante la formación y la diferenciación celular. Entre las radiografías dentales, la panorámica se destaca por ser un examen radiográfico más amplio, que auxilia en el diagnóstico y en la planificación terapéutica de los procesos patológicos de los dientes y de los huesos de la cara. Objetivo: El objetivo de este estudio es determinar la prevalencia de anomalías dentales en radiografías panorámicas de pacientes en tratamiento en el Centro Universitario Católica de Quixadá-CE y en el Centro de Especialidades Odontológicas de Quixeramobim-CE. Métodos: El análisis radiográfico fue realizado por un único investigador y los hallazgos radiográficos fueron escritos en una ficha debidamente desarrollada para este estudio. Resultados: Se analizaron 500 radiografías panorámicas de pacientes que tenían entre 5 y 50 años de edad. De ellos, el $67 \%(\mathrm{n}=333)$ pertenecía al sexo femenino y el $33 \%(\mathrm{n}=167)$ al masculino. Se ha encontrado un total de 1150 anomalías, correspondientes a: dientes no irrompidos $41,7 \%$ $(n=480)$, giroversión $24,4 \%(n=281)$, dilaceración radicular 20,3\% $(n=234)$, microdoncia $6,(N=28)$, agonismo $3,9 \%(n=45)$, dientes supernumerarios $2,4 \%(\mathrm{n}=28)$, taurodoncia $0,3 \%(\mathrm{n}=4)$ y la macrodoncia $0,1 \%(\mathrm{n}==1)$. Las radiografías también fueron divididas por el tipo de arcada ocurriendo $48 \%(\mathrm{n}=307)$ en la mandíbula y 52\% ( $\mathrm{n}=330)$, en la mandíbula. Las anomalías dentales hiperplasitivas se encontraron en el 2,9\% $(n=33)$ de la muestra, las hipoplasiantes en el 10,6\% $(n=122)$ y las heterotópicas en el 86,5\% ( $\mathrm{n}=995)$. De esta forma se concluye que en la población estudiada las mayores predominancias correspondieron al sexo femenino, a la anomalía de desarrollo heterotópica y al diente no irrumpido.

Descriptores: Anomalías Dentárias; Radiografía; Radiografía Panorámica.

\section{INTRODUÇÃO}

Darwin, em 1852, apresentou a teoria da "Seleção Natural", que consiste em adaptar-se ao meio ambiente através de mutações e repassá-las a seus sucessores. A partir desse princípio, muitas pesquisas mostraram a evolução da alimentação e consequentemente da mastigação como um dos fatores coadjuvantes na instalação de anomalias dentárias ${ }^{1}$.

As Anomalias de Desenvolvimento Dentário (ADD) ocorrem devido a distúrbios que acontecem durante a formação e a diferenciação celular. A dentição humana pode apresentar variações de 
tamanho, forma, estrutura, número, cor e formação das estruturas dentárias. A dentição temporária inicia a sua formação intrauterina por volta das 6 semanas e continua o seu desenvolvimento com os dentes permanentes até à adolescência ${ }^{2}$.

Segundo Álvares e Tavano ${ }^{3}$ existem três fases de formação das estruturas do corpo, sendo a primeira de informação genética, em que ocorrem as anomalias hereditárias; a segunda a fase intrauterina, onde ocorrem as anomalias congênitas e a terceira a fase de formação pós-natal, em que ocorrem as anomalias adquiridas. No momento em que o profissional se depara, com qualquer uma dessas alterações, observará diferenças com as condições normais dessas estruturas e, consequentemente, um confronto com anomalias, também denominadas de distúrbios de desenvolvimento.

As anormalidades de tamanho, forma e estrutura são resultados de distúrbios ocorridos durante o estágio de morfodiferenciação e desenvolvimento do dente. É importante o tratamento dessas anomalias, porque elas podem criar alterações dentais da maxila e mandíbula, no comprimento do arco e oclusão. Esses problemas podem criar complicações no tratamento dentário como, por exemplo, a necessidade de um tratamento ortodôntico ${ }^{1}$.

Frequentemente, anomalias de desenvolvimento dentário são identificadas por exames clínicos de rotina e/ou exames radiográficos panorâmicos ${ }^{4}$. A avaliação radiográfica panorâmica da dentadura mista permite visualizar o desenvolvimento intra- ósseo dos germes dentários, bem como o processo de erupção dos dentes permanentes e o de esfoliação dos dentes decíduos, o que possibilita analisar a presença de (ADD), permitindo ao cirurgião-dentista adotar medidas preventivas para um possível comprometimento da estética e/ou função que possam vir a existir ${ }^{5,6}$.

Atualmente a Odontologia está dando mais importância para a necessidade de proporcionar atendimentos especializados à população para que se possa atingir o objetivo de promover e recuperar a saúde ${ }^{7}$. O conhecimento das ADD é fundamental para compreender como elas podem ocasionar má oclusão, deformidades estéticas, acidentes e complicações durante a extração dentária ou tratamento endodôntico. A fim de, diagnosticar essas anomalias, além de observações clínicas, exames como a radiografia são essenciais e desempenham um importante papel no diagnóstico diferencial ${ }^{8}$.

De acordo com Álvares e Tavano ${ }^{3}$, alguns critérios são adotados para classificar as anomalias dentárias que podem ser de ordem funcional, morfológica e histológica, também podem ser causadas por fatores hereditários ou acontecimentos ao longo do seu desenvolvimento como traumatismo ou lesões cariosas nos precursores decíduos.
Segundo essas considerações, as anomalias são divididas em três grandes grupos: anomalias dentárias hiperplasiantes, anomalias dentárias hipoplasiantes e anomalias dentárias heterotópicas.

As anomalias dentárias hiperplasiantes são caracterizadas por um aumento no número de dentes ou tecidos, com alterações estruturais, morfológicas e/ou funcionais ${ }^{9}$. A hiperdontia é um termo que se refere ao desenvolvimento de dentes supranumerários, os quais são descritos como dentes em excesso. Na dentição permanente são mais frequentemente encontrados na região de incisivos superiores, localizados na linha média e na maioria das vezes pequenos, dismorfos e conóides, classificado como mesiodens. O segundo tipo de dentes supranumerários, que podem ser encontrados na dentição permanente são os quarto molares, classificados como; distomolar quando localizados distalmente ao terceiro molar ${ }^{10}$.

A macrodontia, sendo explicada de uma forma mais simplista, envolve dentes de tamanho volumoso, maiores que os considerados normais ${ }^{11}$. A literatura apresenta três tipos de macrodontia: a macrodontia generalizada verdadeira, a macrodontia generalizada relativa, também chamada pseudomacrodontia, e a macrodontia localizada. Quando todos os dentes das duas hemiarcadas são maiores do que o normal designa-se a alteração por macrodontia generalizada verdadeira. A macrodontia generalizada relativa ou pseudomacrodontia é o termo usado para descrever uma situação em que a mandíbula e/ou a maxila se apresentam menores do que o normal, mas os dentes apresentam dimensões normais. A macrodontia localizada é observada, por exemplo, em casos de hipertrofia hemifacial unilateral, resultando num hiperdesenvolvimento das coroas do lado afetado ${ }^{12}$.

Em circunstâncias normais, a maioria dos molares tem uma coroa que representa $1 / 3$ da longitude do dente e a raiz. A taurodontia é uma anomalia morfológica que se caracteriza por um aumento do corpo do dente e um encurtamento das raízes, deslocando a furca para apical, constituindo assim uma alteração de desenvolvimento dos dentes na qual o formato anatómico dentário se encontra alterado ${ }^{13}$.

As anomalias dentárias hipoplasiantes têm por característica a diminuição ou desenvolvimento incompleto de dentes ou tecidos, com alterações estruturais, morfológica e/ou funcionais ${ }^{3}$.

Agenesia é a ausência completa ou parcial de um órgão e seu primórdio embriológico. A agenesia dentária é uma alteração do desenvolvimento tendo como etiologia principal a hereditariedade, é considerada uma das anomalias congênitas mais frequentes nos seres humanos caracterizando-se pela ausência de um ou mais dentes e comprovado através de exame radiográfico. 
A etiologia da agenesia é multifatorial, inclui-se uma pré-disposição genética, fatores externos, radiação e síndromes e ocorre em $25 \%$ da população aproximadamente. Para o diagnóstico preciso, faz-se necessário o uso da radiografia a qual exerce um papel fundamental para comprovar a ausência de dentes numa idade em que deveriam estar presentes ${ }^{14}$.

Microdontia é uma condição na qual os dentes são anormalmente pequenos. A forma localizada ocorre em um ou alguns dentes, uni ou bilateral, afetando total ou parcialmente. $\mathrm{Na}$ microdontia total teremos uma diminuição da extensão total do dente, coroa e raiz, na parcial há uma diminuição apenas de uma parte do dente, coroa ou raiz. A forma mais comum encontrada é a forma conóide, em cujas faces, mesial e distal, convergem para incisa ${ }^{15}$.

As anomalias dentárias heterotópicas são caracterizadas pela erupção e/ou posição dos dentes fora do seu local habitual, ou ainda pelo deslocamento e desenvolvimento dos tecidos dentários com perda da relação normal entre eles ${ }^{3}$.

A dilaceração é o resultado da anomalia de desenvolvimento que provoca uma mudança abrupta na inclinação axial entre a coroa e a raiz de um dente. Durante a odontogênese, qualquer fator que possa mudar o metabolismo e as condições físicas ao redor do germe dentário pode perturbar o seu desenvolvimento ${ }^{8}$.

Existe uma divergência na literatura quanto à terminologia mais apropriada para designar a situação de inclusão dental. Considera-se inclusos os dentes que, chegada sua época fisiológica de erupção, permanecem imersos no interior dos tecidos, devido a impedimentos físicos ou patológicos. Os dentes inclusos podem estar semiinclusos quando apresentam apenas parte de sua coroa dental exposta ao meio bucal. E ainda podem estar impactados quando seu trajeto de erupção estiver obstruído por um fator mecânico ou patológico específico ${ }^{16}$.

Dentes girovertidos podem ser classificados quando a superfície mesial ou a superfície distal dos dentes estiver desviada a partir da linha da arcada dentária ${ }^{17}$.

De acordo com Yamada ${ }^{18}, 5 \%$ da população já nascem com alguma anomalia hereditária e cerca de $60 \%$ destas anomalias envolvem os dentes do maxilar superior, ou na face. Certas anomalias de forma, como lacerações, dente supranumerário, dente invaginado e taurodontismo, não causam alterações significativas a saúde bucal do paciente, mas exigem uma maior atenção em seu tratamento e acompanhamento.

Diversos fatores são determinantes e dificultam o tratamento de dentes com anomalias. Com isso, torna-se relevante a análise minuciosa da radiografia panorâmica, pois através dela podemos identificar diversas anomalias, que não apresentam nenhuma sintomatologia clínica no paciente, pois através da mesma torna-se possível elaborar um plano de tratamento e acompanhamento adequado. Sendo assim, o objetivo desse estudo é determinar a prevalência de anomalias dentárias em radiografias panorâmicas de pacientes avaliados para tratamento no Centro Universitário Católica de Quixadá-CE e no Centro de Especialidades Odontológicas (CEO) do município de Quixeramobim-CE.

\section{MATERIAL E MÉTODO}

O presente trabalho foi aprovado pelo Comitê de Ética do Centro Universitário Católica de Quixadá com o Parecer de número 1.990.5046, atendendo os termos da Resolução 466/12 do Conselho Nacional de Saúde; constituiu-se de abordagem descritiva, quantitativa transversal e retrospectivo, apresentando a prevalência de anomalias, radiograficamente, de pacientes entre $5 \mathrm{e}$ 50 anos. Foram analisadas 500 radiografias panorâmicas de pacientes, de ambos os sexos, no período de 2013 a 2017, atendidos no Centro Universitário Católica de Quixadá-CE e no Centro de Especialidades Odontológicas de Quixeramobim-CE.

As radiografias selecionadas, consideradas ideais, apresentaram o mínimo de distorção, o máximo de nitidez e grau médio de contraste e densidade. Foram excluídas as radiografias com manchamento, devido a erros no processamento, ou com baixa nitidez de contraste e densidade.

Realizou-se a divisão em arcada superior e inferior. As anomalias dentárias foram classificadas segundo Alvares e Tavano ${ }^{3}$, sendo estudadas nas radiografias panorâmicas apenas algumas alterações dentárias de desenvolvimento hiperplasiantes, hipoplasiantes e heterotópicas. As radiografias analisadas também foram separadas por sexo e faixa etária mais prevalente.

A análise radiográfica foi realizada por um único pesquisador e os achados radiográficos foram escritos em uma ficha devidamente desenvolvida para este estudo. O ambiente utilizado para o procedimento de interpretação radiográfica apresentou condições ideais de iluminação, ou seja, em uma sala cuja única luz foi a do negatoscópio e com o auxílio de uma lupa.

Após a coleta dos dados transcritos na ficha e tabulação dos resultados através do programa Microsoft Excel 2016. Os resultados foram apresentados em gráficos e tabelas, devidamente comentados.

\section{RESULTADOS}

A amostra da pesquisa aleatória consistiu de 500 radiografias panorâmicas. Os pacientes apresentaram idades variando de 5 a 50 anos (média 
de idade $=25,32$ ) e uma prevalência maior na faixa etária entre 15 e 30 anos, dos quais $67 \%(n=333)$ eram do sexo feminino e $33 \%$ do sexo masculino $(\mathrm{n}=167)$. Foram excluídas 15 radiografias devido à baixa qualidade apresentada. Sendo assim ficamos com uma amostra final de 485 radiografias selecionadas (Gráfico 1).

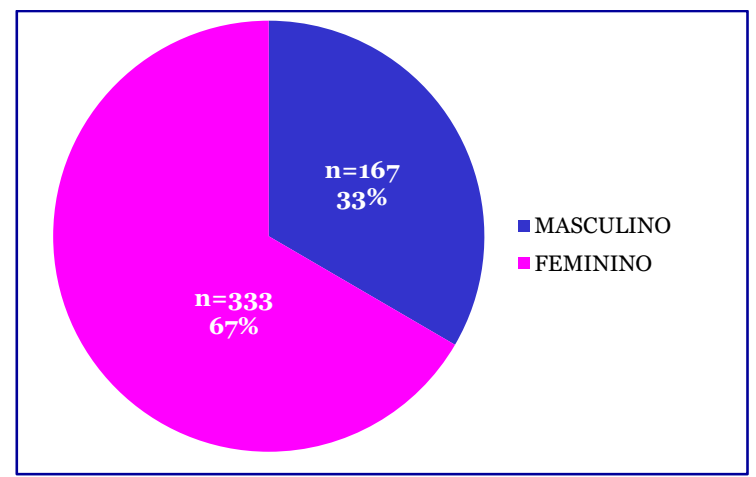

Gráfico 1: Distribuição das radiografias panorâmicas segundo o sexo.

Das 485 radiografias panorâmicas analisadas, apenas 80 não apresentaram nenhum tipo de anomalia. Em $92,78 \%$ das radiografias analisadas $(\mathrm{n}=405)$ foram identificados: dentes não irrompidos $41,7 \% \quad(n=480)$, giroversão $24,4 \% \quad(n=281)$, dilaceração radicular $20,3 \%(\mathrm{n}=234)$, microdontia $6,7 \% \quad(\mathrm{n}=77)$, agenesia $3,9 \% \quad(\mathrm{n}=45)$, dentes supranumerários $2,4 \% \quad(\mathrm{n}=28)$, taurodontia $0,3 \%$ $(\mathrm{n}=4)$ e a macrodontia $0,1 \%(\mathrm{n}=1)$ totalizando 1150 anomalias (Gráfico 2). Não foi encontrado nenhum tipo de anomalia na dentição decídua. Os números de anomalias não coincidem com o número de radiografias analisadas devido à presença de mais de uma anomalia em um mesmo paciente.

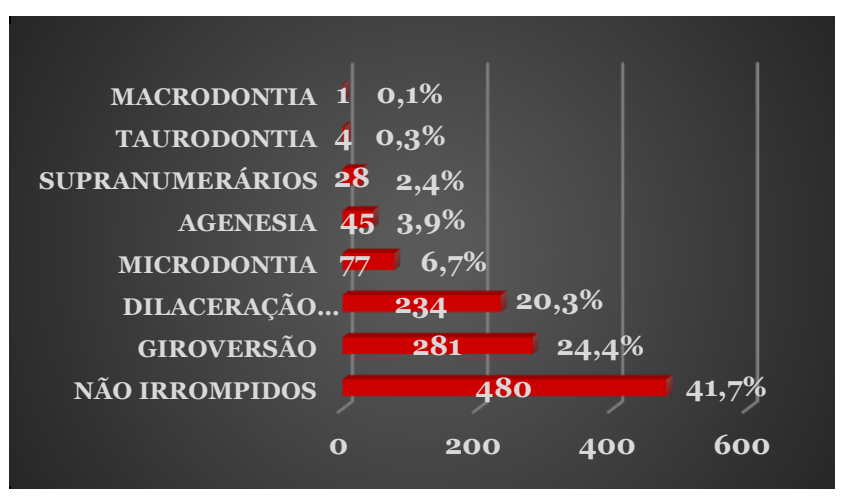

Gráfico 2: Distribuição numérica e percentual de anomalias encontradas nas radiografias panorâmicas.

Observou-se que o acometimento pelas ADD em $48 \%(\mathrm{n}=307)$ das maxilas avaliadas, onde foram encontradas um total de $49 \%(\mathrm{n}=563)$ anomalias. $\mathrm{Na}$ maxila o dente 28 foi o mais acometido e a anomalia mais prevalente foram os dentes não irrompidos. Já na mandíbula houve uma ocorrência de $52 \%(\mathrm{n}=330)$, em que foram encontradas $51 \%(\mathrm{n}=587)$ anomalias e o dente mais acometido foi o dente 48 e a anomalia mais prevalente também foram os dentes não irrompidos (Gráfico 3).
As anomalias encontradas foram divididas também por tipo de anomalia de desenvolvimento. Das anomalias dentárias hiperplasiantes foram encontradas 2,9\% $\quad(n=33)$ anomalias dentárias hipoplasiantes $10,6 \%(n=122)$ e anomalias dentárias heterotópicas 86,5\% (n=995) (Gráfico 4).

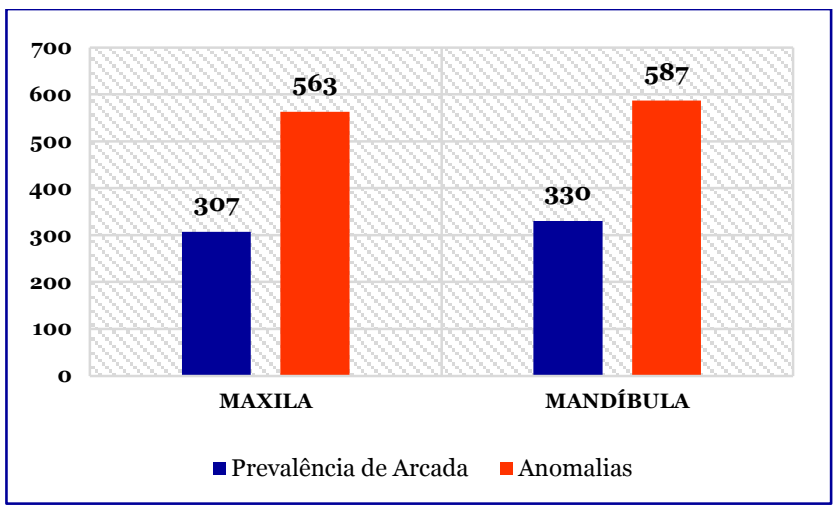

Gráfico 3: Número de arcadas acometidas e de anomalias por arcada.

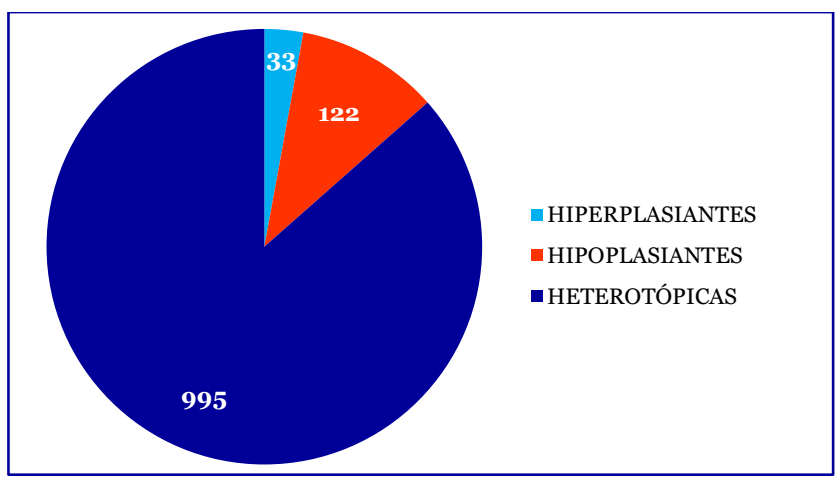

Gráfico 4: Distribuição de frequência de acordo com o tipo de anomalia de desenvolvimento encontrada.

\section{DISCUSSÃO}

As ADD podem ocorrer tanto em função de fatores locais como sistêmicos podendo se iniciar antes ou após o nascimento, consequentemente, afetando dentes decíduos ou permanentes ${ }^{19}$. Existem poucos trabalhos como este, que analisam ADD com relação à localização, idade e sexo, em indivíduos adultos e crianças. Talvez isso possa ser explicado em função da dificuldade de se compararem os resultados encontrados de anomalias em decorrência da diversidade de variáveis analisadas, tamanho diferente das amostras e interpretação individualizada de cada pesquisador.

O conhecimento das anomalias dentárias é de primordial importância para elaboração do plano de tratamento de pacientes atendidos na odontologia e para prevenção de complicações posteriores, dentre as quais: atraso no irrompimento de dentes permanentes, desvio na trajetória eruptiva, rotação, dentre outros, podem ocasionar problemas oclusais ${ }^{20}$.

Menini et al. ${ }^{13}$ observaram em seu estudo que $56.84 \%(\mathrm{n}=457)$ dos participantes pertenciam ao sexo feminino e $43.16 \%(n=347)$ ao masculino. Estes 
dados vão ao encontro do presente estudo que apresentou uma maior prevalência do sexo feminino $67 \%(\mathrm{n}=333)$ e do sexo masculino 33\% ( $\mathrm{n}=167)$. Vale ressaltar que houve um número maior de pacientes do sexo feminino devido a uma maior procura de atendimento odontológico por mulheres.

Carneiro ${ }^{1}$ mostrou em seu estudo que houve uma maior ocorrência de anomalias na mandíbula em uma proporção de 9:7. Estando de acordo com o presente estudo onde a mandíbula foi mais acometida que a maxila representando $52 \%(n=330)$ da amostra.

No presente estudo, a ADD mais prevalente tanto na arcada maxilar quanto na mandibular foi dente não irrompido, seguida de giroversão, dilaceração radicular, microdontia, agenesia, dentes supranumerários, taurodontia e macrodontia (Gráfico 2).

Menini et al. ${ }^{13}$ analisaram em seu estudo 804 radiografias e encontraram 232 anomalias. No presente estudo foram analisadas 485 radiografias e encontradas 1150 anomalias. É de grande importância ressaltar que o número elevado de anomalias encontradas neste estudo pode estar relacionado à faixa etária do público atendido, que apresentam uma idade entre 15 e 30 anos e apresentaram os terceiros molares não irrompidos.

$\mathrm{Na}$ cavidade bucal é muito comum encontrarmos dentes irrompidos, Costa et al. ${ }^{16}$ encontrou em seu estudo 18,7\% ( $n=265)$ dentes não irrompidos dados que diferem do presente estudo que apresentou $41,7 \%$ da amostra $(n=480)$. O elemento dentário mais acometido por essa anomalia foi o 48 , mas os terceiros molares 38, 28 e 18 obtiveram também uma alta prevalência.

Colombo et al. ${ }^{21}$ obtiveram em seus resultados uma correspondência clínica/radiográfica de $51,4 \%$ de dentes girorvertidos. Os caninos inferiores foram os dentes mais acometidos pelas anomalias dentárias nesse estudo. Isto provavelmente é consequência do fato destes dentes sofrerem alterações de posição (giroversões parciais ou totais, no irrompimento) graças à localização na arcada e à cronologia de irrompimento ${ }^{7}$. Esses dados corroboram com os achados do presente estudo que obteve uma ocorrência de $24,4 \quad(n=281)$ para a giroversão e dentes mais acometidos foram os caninos inferiores.

Guttal et al. ${ }^{22}$ em seu estudo das raízes constituíram $22,5 \%$ das anomalias. Teixeira et al. ${ }^{23}$ concluíram em seu estudo que a anomalia de forma mais observada foi à dilaceração radicular $57.76 \%$. No presente estudo observou-se que a dilaceração radicular esteve presente em 20,3\% ( $n=234)$ dentes da amostra.

Os dentes comumente afetados são os Incisivos e terceiros molares. A prevalência desta condição varia de $0,8 \%$ a $8,4 \%$ em várias populações. Quando os incisivos laterais são afetados, há uma redução no diâmetro mesiodistal e convergência para a borda incisal, referidos como pinos em forma de incisivos. A condição é em grande parte de origem genética ${ }^{8}$.

Menini et al. ${ }^{13} \mathrm{em}$ seu estudo mostraram que a microdontia representou $12.93 \%$ da amostra e somente em indivíduos maiores de 12 anos de idade. Isso provavelmente se deve ao fato de que as microdontias foram encontradas nos terceiros molares. Neste estudo, a microdontia constituiu 6,7\% $(n=77)$ da amostra estando coerente com os achados encontrados na literatura e houve uma ocorrência apenas em pacientes adultos.

Menini et al. ${ }^{13}$ em seu estudo observaram $15,09 \%(\mathrm{n}=35)$ de agenesias. Já Guttal et $\mathrm{al}^{22}$ encontraram uma proporção foi de $10.06 \%$ de agenesia em seu estudo. O presente trabalho está de acordo com o estudo de Polder et al. ${ }^{24}$ que em uma revisão sistemática dos valores na população mundial e constataram uma variação em torno de $2.2 \%$ a $10.1 \%$, valor este muito próximo ao aqui encontrado e dentro das proporções, levando em consideração que o percentual de agenesia do presente trabalho foi de $3,9 \%(n=45)$.

A hiperdontia é encontrada mais comumente em crianças ou adolescentes. A sua prevalência varia de $0,1 \%$ para $3,8 \%{ }^{25}$ achados que corroboram com este estudo que representou $2,4 \%(n=28)$ da amostra.

A taurodontia mostra-se mais prevalente em mulheres do que em homens, sendo a maxila mais afetada do que a mandíbula ${ }^{7}$. Dados que vão de encontro ao presente estudo, onde os dentes com taurodontia foram encontrados apenas na maxila e correspondendo $0,3 \%(n=4)$ da amostra.

No presente estudo, apenas $0,1 \% \quad(n=1)$ da amostra apresentou casos de macrodontia, possivelmente em função de sua baixa prevalência, relatada como de $0,2 \%$ nos estudos de Ezoddini et al. ${ }^{26}$.

\section{CONCLUSÃO}

De acordo com a proposta deste trabalho, pode-se concluir que, na população estudada, o sexo mais prevalente foi o feminino representando $67 \%$ da amostra. A anomalia de desenvolvimento Heterotópica foi a mais predominante $86,5 \%$ $(\mathrm{n}=995)$, sendo o dente não irrompido responsável por $41,7 \%$. A girorversão correspondeu a $24,4 \%$ seguida da dilaceração radicular representando $20,3 \%$ da amostra.

\section{REFERÊNCIAS}

1. Carneiro GV. Estudo radiográfico da prevalência de anomalias dentárias por meio de radiografias panorâmicas em diferentes faixas etárias [tese]. Programa de Pós-graduação em Saúde e Desenvolvimento na Região Centro-Oeste; 2014.

2. Seabra M, Macho V, Pinto A, Soares D, Andrade C. A importância das anomalias dentárias de 
desenvolvimento. Acta Pediatr Port. 2008;39(5):195-200.

3. Álvares LC, Tavano O. Curso de radiologia em odontlogia. São Paulo: Santos; 2009.

4. Paula, AFB, Ferrer KJN. Prevalência de agenesia em uma clínica ortodôntica de Goiânia. RGO. 2007;55(2):149-53.

5. Gartner CF, Goldenberg FC. A importância da radiografia panorâmica no diagnóstico e no plano de tratamento ortodôntico na fase da dentadura mista. Rev Odonto. 2009;17(33):102-9.

6. Barbieri AA. A importância da radiografia panorâmica como instrumento auxiliar às práticas clínica e odontolegal [dissertação]. São José dos Campos: Universidade Estadual Paulista; 2011.

7. Santos MR, Olibeira KL, Fonte JBM, Hora IAA, Takeshita WM, Melo MFB. Prevalência De Alterações Dentárias Em Pacientes Com Síndrome De Down Avaliados Por Meio De Radiografia Panorâmica. Rev Odontol Univ Cid São Paulo. 2014;26(2):112-18.

8. Pereira AC, Nishiyama CK, Pinto LC. Anomalias dentárias em indivíduos com fissura transforame incisivo unilateral e o tratamento endodôntico. RFO UPF. 2013;18(3):328-34.

9. Scarpim MFPA, Nunes VS, Cerci BB, Azevedo LR, Tolazzi AL, Grégio AMT et al. Prevalência de anomalias dentárias em pacientes avaliados para tratamento ortodôntico: estudo retrospectivo. Clin Pesq Odontol. 2006;2(3):203-12.

10.Torres PF, Simplício AHM, Luz ARCA, Lima MDM, Moura LFAD, Moura MS. Anomalias dentárias de número em pacientes ortodônticos. Rev Odontol UNESP. 2015;44(5):280-84

11.Girondi JR, Fenyo-Pereira M, Campos PSF, Panella J. Estudo da prevalência das anomalias dentárias de desenvolvimento em dada população com o uso de radiografi as panorâmicas. Rev Odont Univ Cid São Paulo. 2006;18(1):15-21.

12. Canoglu E, Canoglu H, Aktas A, Cehreli ZC. Isolated bilateral macrodontia of mandibular second premolars: a case report. Eur J Dent. 2012; 6(3):330-34.

13.Menini AAS, Silva MC, Iwaki LCV, Takeshita WM. Estudo radiográfico da prevalência de anomalias dentárias por meio de radiografias panorâmicas em diferentes faixas etárias. Rev Odontol Univ Cid São Paulo. 2012;24(3):170-77.

14.Barbosa DFM, Cruz CM, Crepaldi MV, Oliveira BLS. Agenesias múltiplas, planejamento e hereditariedade. Rev Faipe. 2016;6(2):14-27.

15.Inoue T, Saito M, Nishimura F, Miyazaki T. Three-dimensional representation of microdontia of the maxillary third molar. Clin Case Rep. 2017;5(4):547-48.

16. Costa MA, Oliveira AEF, Costa JF, Silva RA, Lopes FF, Silva APB. Incidência das posições anatômicas e agenesia dos terceiros molares em estudantes de São Luís, Maranhão. Pesqui bras odontopediatria clin integr. 2010;10(3):399-403.

17.King NM, Wong WL, Wong HM. Caries experience of Chinese children with cleft lip and palate. Cleft Palate Craniofac J. 2013;50(4):448-55.

18. Yamada N. Radiographic abnormalities in genetic diseases. Dent Outl. 1983;62(1):71-8.

19.Guttal KS, Naikmasur VG, Bhargava P, Bathi RJ. Frequency of developmental dental anomalies in the Indian population. Eur J Dent. 2010;4(3):263-69.

20.Garib DG, Alencar BM, Ferreira FV, Ozawa TO. Anomalias dentárias associadas: o ortodontista decodificando a genética que rege os distúrbios de desenvolvimento dentário. Dental Press J Orthod. 2010;15(2):138-57.

21. Colombo LT, Paulon SS, Coclete GA, Coclete GEG, Gaetti Jardim Junior E, Castro AL. Giroversão dental presente ou ausente em radiografias panorâmicas. Arch Health Invest. 2013;2(Esp 2):224.

22.Teixeira VP, Martins MAT, Lascala CA, Marques MM, Rossi JM, Missawa GTM et al. Estudo de anormalidades dentárias de desenvolvimento em pacientes em tratamento ortodôntico Study of development dental abnormalities in orthodontic patients. Rev Inst Ciênc Saúde. 2008;26(4):454-57.

23.Polder BJ, Van't Hof MA, Van der Linden FP, Kuijpers-Jagtman AM. A meta-analysis of the prevalence of dental agenesis of permanent teeth. Community Dent Oral Epidemiol. 2009; 32(3):217-26.

24.Mafra RP, Vasconcelos RG, Vasconcelos MG, Queiroz LMG, Barboza CAG. Desenvolvimento dental: aspectos morfogenéticos e relações com as anomalias dentárias do desenvolvimento. Rev bras odontol. 2012;69(2):232-37.

25.Ezoddini AF, Sheikhha MH, Ahmadi H. Prevalence of dental anomalies: a radiographic study. Community Dent Health. 2007; 424(3):140-44.

\section{CONFLITO DE INTERESSES}

Os autores declaram não haver conflitos de interesse.

\section{AUTOR PARA CORRESPONDENCIA}

\section{Roque Soares Martins Neto}

roquemartinssn@outlook.com

Submetido em 23/09/2018

Aceito em 06/02/2019 